\title{
DIAGNÓSTICO DA NEUROCISTICERCOSE ATRAVÉS DA TOMOGRAFIA AXIAL COMPUTADORIZADA DE CRÂNIO: ANÁLISE DE 2030 CASOS
}

\author{
WANDER MIGUEL TAMBURUS" \\ ELIANA CHRISTINA DE FIGUEIREDO WANDERLEY" \\ FAHD HADDAD \\ LAMARTINE CORREA DE MORAES JÚNIOR" \\ DAMÁCIO RAMON KAIMEN MACIEL" \\ AVELINO JOSÉ SOARES NARCISO² \\ MAURICIO MICHALAK SENDESK ${ }^{3}$
}

\begin{abstract}
TAMBURUS, W.M.; WANDERLEY, E.C.de F.; HADDAD, F.; MORAES JUNIOR, L.C.de; MACIEL, D.R.K.; NARCISO, A.J.S.; SENDESKI, M.M. Diagnóstico da neurocisticercose através da tomografia axial computadorizada de crânio: análise de 2030 casos. Semina: Ci.Biol/Saúde, Londrina, v. 16, n. 2, 19. 317-320, jun. 1995.
\end{abstract}

\begin{abstract}
RESUMO: O diagnóstico da neurocisticercose através da tomografia axial computadorizada (TAC) de crânio es seguro, confiável e nåo invasivo, possuindo sensibilidade superior às reaçöes imunológicas no LCR e identificando as várias fases de evoluçăo da doença. Nosso objetivo foi estudar as caracteristicas da populaçăo em que se diagnosticou neurocisticercose no Instituto de Tomografia Axial Computadorizada (I. T.A.C.) de Londrina, bem como os aspectos tomográficos encontrados. Foram revisadas todas as TAC de cranio realizadas no I.T.A.C. de Londrina entre setembro de 1986 e maio de 1994, sendo analisados os exames de pacientes em que se diagnosticou neurocisticercose. Estudou-se a presença de calcificaçסes, cistos ou vesiculas, granulomas e hidrocefalia. Analisouse também a idade, sexo e procedência de zona rural ou urbana. Holsve diagnóstico de neurocisticercose em 2030 pacientes. A maioria (47,6\%) possuia idade entre 20 e 40 anos; 886 (43,7\%) eram do sexo masculino, e 1144 $(56,3 \%)$ do sexo feminino. Apenas 85 pacientes procediam de zonarural. Dentre os achados tomográficos, houve 983 calcificaçס̃es únicas (48,4\%), 873 calcificações múltiplas $(43,0 \%), 199$ cistos ou vesículas (9,8\%) e 121 granulomas (5,9\%). O achado de hidrocefalia esteve presente em 135 casos (6,7\%), em 86 pacientes havia cisticercos em diferentes estágios de evolução. Os resultados são discutidos a luz da literatura disponivel.
\end{abstract}

PALAVRAS CHAVE: Tomografía computadorizada; Neurocisticercose.

\section{1 - INTRODUÇÃO}

A neurocisticercose é a afeç̧ão parasitária mais comum do sistema nervoso central, acometendo até $4 \%$ da população em áreas endêmicas (SOTELO et al.,1985), e decorre da infestação do sistema nervoso central pela forma larval (Cysticercus cellulosae) da Taenia solium. A infestação ocorre após ingestão de ovos viáveis (provenientes de eliminação humana via fecal, de contato fecal-oral ou auto-infestação), ou através de retro-peristaltismo trazendo proglotides ao estômago (SCHARF, 1988).

A neurocisticercose acomete todas as faixas etárias, mas o diagnóstico se faz mais frequentemente na terceira a quarta décadas de vida. Ambos os sexos são acometidos em frequência semelhante, havendo predominio do sexo masculino em alguns relatos (CHEQUER \& VIEIRA, 1990; SOTELO et al.,1985). Quanto à localização do cisticerco, a neurocisticercose pode ser classificada em forma meningea, forma ventricular, forma parenquimatosa e forma mista (BYRD et al., 1982:MERVIS \& LOTZ,1980). O acometimento medular incomun, e usualmente caracteriza-se por cistos no espaço sub-aracnóide, podendo haver aracnoidite (SOTELO et al., 1985). A forma racemos: uma variante de acometimento meningeo, consite na proliferaç๐o das membranas do cisticerco assumindo uma forma de cacho de uva, usualmente nas meninges basais (NASH \& NEVA, 1984).

O quadro clínico da doença é pleomórfico, vaniando a apresentação clinica conforme a localização. o tamanho, o número e o estágio de desenvolvimento dos cisticercos, conforme a magnitude da resposta inflamatoria do hospedeiro ao parasita. Acredita-se que uma proporção considerável dos acometimentos seja assintonåtica (MACHADO et al., 1990; CHEQUER \& VIEIRA,1990; GUERREIRO et a!., 1989; MINGUETTI \& FERREIRA,1983; SCHARF,1988; SOTELO et al.,1985).

A romografia axial computadorizada (TAC) de crânio constitui o principal método diagnóstico utilizado para neurocisticercose: é segura, confiável e não invasiva, possibilitando identificar a topografia e a fase de desenvolvimeto do parasita. A TAC possu sensibilidade superior às reações imunológicas no liquido

\footnotetext{
Trabalho realizado no instituto de Tomografia Axial Computadorizada (ITAC) de Londrina Departamento de Cilnica Cirúrgica do Centro de Ciências da Saúde (CCS) da Universidade Estadual de Londrina (UEL). Av Robert Koch, n.60, Londrina, PR., CEP 86038-440.

1 Departamento de Cirnica Cirúrgica do CCS/Universidade Estadual de Londrina.

2 Médico Residente em Neurocirurgia do Hospital Universitário Regional do Norte do Paraná da UEL.

3 Acadêmico do Curso de Medicina da Universidade Estadual de Londrina.
}

Semina Ci. Biol./Saúce, v. 16, n. 2, p. 317.320 
cefalorraquidiano comumente utilizadas para a neurocisticercose (MIRANDA,1993; SOTELO et al.,1985; TAKAYANAGUI,1990): a precisão diagnóstica da TAC pode chegar a $97 \%$ quando existe correlação clínica-radiológica (RODRIGUEZ-CARBAJAL\&BOLEAGA-DURAN, apud ALMEIDA-PINTO et al.,1988).

A TAC adquire especial importância na avaliação da forma parenquimatosa da doença, sendo que o aspecto encontrado ao exame depende da fase de evolução do parasita (MERVIS \& LOTZ,1980). O cisticerco jovem, ainda vivo, aparece sob a forma de cisto, de tamanho variável, sem área de reforço após injeção de contraste; o número de cistos evidenciados varia conforme o grau de infestação do paciente. Ao iniciar-se a degeneração do parasita, o cisto diminui de tamanho, aparecendo circundado por um reforço homogêneo de 1 a 2 cm durante a fase contrastada; é a fase de nódulo, na qual a TAC não contrastada pode ser normal. Após esta fase inicia-se a deposição de sais de cálcio no local, levando à fase de calcificação: imagens de 2 a $6 \mathrm{~mm}$, redondas ou ovais, sem captação de contraste, ocorrendo geralmente na junção da substância branca com cinzenta (ALMEIDA-PINTO et al.,1988; BYRD et al., 1982; GUERREIRO et al., 1989; RAWLINGS et al., 1989; TAKAYANAGU1, 1990).

O intervalo entre a morte e a calcificação do cisticerco é estimado entre 1 a 7 anos (BYRD et al., 1982; MINGUETTI \& FERREIRA, 1983; TAKAYANAGUI, 1990), podendo ser de apenas três meses (ALMEIDA-PINTO et al.,1988). Cisticercos em diferentes fases de desenvolvimento podem ser encontrados no mesmo paciente, visto que a infestação pode ocorrer num processo contínuo. $O$ aspecto tomográfico predominantemente encontrado é a calcificação (ALMEIDA-PINTO et al.,1988; CHEQUER \& VIEIRA, 1990; GUERREIRO et al.,1989; MINGUETTI \& FERREIRA, 1983; SOTELO et al.,1985; TAKAYANAGUI,1990).

O valor da TAC na avaliação das formas meníngeas e ventriculares é menor em relação à forma parenquimatosa. Entretanto, áreas não específicas de captação aumentada de contraste em meninges basais podem ser evidenciadas na forma meníngea, e áreas de infarto cerebral (em decorrência de arterite associada à inflamação meníngea) podem também ser encontradas, bem como a presença de hidrocefalia. Nestes casos, o diagnóstico é feito através do envolvimento parenquimatoso associado (ALMEIDA-PINTO et al.,1988). A hidrocefalia pode ser decorrente tanto de obstrução (SCHARF,1988) como de envolvimento meníngeo agudo ou crônico (ALMEIDA-PINTO et al.,1988; SOTELO et al., 1985).

O diagnóstico diferencial tomográfico deve incluir toxoplasmose, hidatidose, tuberculose, abcesso cerebral, granuloma micótico, tumores calcificados, metástases, astrocitoma com apresentação cística, porencefalia e esclerose tuberosa, principalmente quando se trata de lesões calcificadas únicas (ALMEIDAPINTO et al., 1988).

Alguns estudos têm reportado as características clínico-epidemiológicas da neurocisticercose em localidades específicas (ARRUDA et al., 1990; CHEQUER \& VIEIRA, 1990); nosso objetivo foi avaliar as caracteristicas da população em que se diagnosticou neurocisticercose no Instituto de Tomografia Axial Computadorizada de Londrina, bem como os aspectos tomográficos encontrados.

\section{METODOLOGIA}

O Instituto de Tomografia Axial Computadorizada (ITAC) de Londrina atende à região de Londrina e cidades vizinhas, realizando em média 150 TAC de crânio por mês. Foram revisadas todas as TAC de crânio efetuadas pelo ITAC no período compreendido entre setembro de 1986 e maio de 1994, sendo analisados os exames de pacientes em que se diagnosticou neurocisticercose. Discriminaram-se as formas calcificada, vesicular e granulomatosa, sendo ainda computada a presença de hidrocefalia. Analisaram-se também alguns dados clínicos desses pacientes (idade, sexo, procedência de zona rural ou urbana).

\section{RESULTADOS}

O diagnóstico de neurocisticercose se fez em 2030 pacientes; 886 (43.7\%) desses pacientes eram do sexo masculino, e $1144(56.3 \%)$ do sexo feminino. Houve predominância do sexo heminino em lodos os grupos etários (Gráfico 1). A maioria dos pacientes roi procedente da zona urbana (1945 casos), e a minoria da zona rural (85 casos). A idade minima encontrada foi de 12 meses, e a máxima de 85 anos.

\section{GRÁFICO 1 - DISTRIBUIÇÃO DOS PACIENTES SEGUNDO GRUPO ETÁRIO E SEXO}

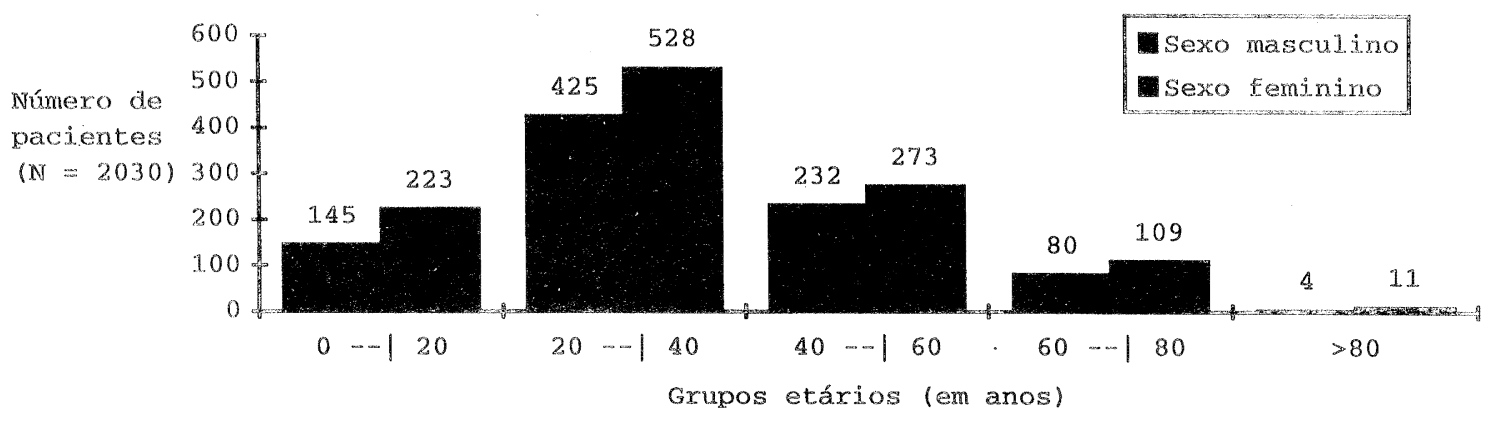

Semina Ci. Biol/Sauide, v. 16, n. 2, p. $317-320$ 
Predominaram nos achados tomográficos as calcificações únicas (presentes em 983 casos - $48.4 \%$ do total) e as calcificaçס̃es múltiplas (873 casos - $43.0 \%$ do total); 0 achado de hidrocefalia esteve presente em 135 casos (6.7\% do total), e cistos ou vesiculas foram encontrados em 199 casos ( $9.8 \%$ do total). O achado de granuloma se fez em 121 casos (5.9\% do total), e em 86 pacientes havia cisticercos em diferentes estágios de evolução (Tabela 1).

\section{TABELA 1 - RELAÇÃO DOS ACHADOS TOMOGRÁFICOS.}

$\begin{array}{ll}\text { Calcificações únicas } & 983 \\ \text { Calcificações múltiplas } & 873 \\ \text { Cistos } & 199 \\ \text { Hidrocefalia } & 135 \\ \text { Granulomas } & 121\end{array}$

\section{4 - DISCUSSÃO}

Predominou em nossa casuística o sexo feminino, o que contrasta com os dados reportados na literatura; de fato, relata-se ou equilíbrio na distribuição dos sexos ou discreta predominância do sexo masculino (cuja explicação sugerida seria o predomínio da atividade externa neste sexo [CHEQUER \& VIEIRA,1990]: em tal série, a maioria dos pacientes era proveniente de zona rural).

A análise dos grupos etários mostra dados semelhantes aos reportados na literatura: a maioria dos pacientes (47,6\% do total) possuía idade entre 20 e 40 anos. Pelo fato de tratar-se da faixa etária de maior produtividade ao trabalho, a possibilidade de comprometimento do rendimento económico de uma população com alta prevalência de neurocisticercose não deve ser desprezada.

Houve, em nossa série, exames sugestivos de neurocisticercose em crianças de até 12 meses de idade; nestes grupos etários, a possibilidade de infestação pelo Cysticercus cellulosae é muito baixa, devendo ser feito com precisão o diagnóstico diferencial entre neurociticercose e outras patologias (principalmente infecções congênitas).

Obtivemos, dentre os achados tomográficos, predominio das calcificações. A calcificação é a fase de sequiela da doença, na qual o cisticerco está morto, e persiste definitivamente no interior do tecido nervoso: a possibilidade de existir sintomatologia nestes casos depende do tipo e localização da lesão causada pelo cisticerco em seu processo de instalação e degeneração. As fases vesicular (na qual o parasita está ainda vivo) e granulomatosa (quando ocorre a degeneração do cisticerco) são transitórias, e portanto naturalmente menos prevalentes. Nossos resultados correspondem ao processo evolutivo da neurocisticercose, e, exceto pela porcentagem de casos com hidrocefalia associada (menor do que o reportado em outros relatos [ANTONIUK et al.,1991: JOZZOLINO,1984; MACHADO et al.,1990; SCHARF, 1988; SOTELO et al., 1985]), corroboram os dados da literatura.

A tomografia axial computadorizada de crânio constitui método diagnóstico seguro e não invasivo para a neurocisticercose, $e$, identificando suas variadas apresentações (calcificaçōes, vesiculas, granulomas hidrocefalia), representa acessório fundamental para avaliação do tratamento utilizado.

TAMBURUS, W. M.; WANDERLEY, E. C. de F.; HADDAD, F.; MORAES JUNIOR, L. C. de; MACIEL, D. R. K.; NARCISO, A. J. S.; SENDESKI, M. M. Diagnosis of neurocysticercosis using computed tomography: analysis of 2030 cases. Semina: Ci.Biol/Saúde, Londrina, v. 16, n. 2, p. 317-320, Jun. 1995.

ABSTRACT: The diagnosis of neurocysticercosis using computed tomographic (CT) scans is safe, reliable and noninvasive; it identifies the various phases of the disease, and is more sensitive than immunologic tests in CSF. Our objective was to evaluate the population in wich neurocysticercosis was diagnosed in the Instituto de Tomografia Axial Computadorizada (I.T.A.C.) of Londrina, as well as the appearances found in the exams. We reviewed all the skull CT scans performed in the I.T.A.C. of Londrina between september 1986 and may 1994, and studied those in wich the diagnosis of neurocysticercosis was made. The appearances of calcification, vesicles, granulomas and hydrocephalus were analysed, as well as the age, sex and origin from rural or urban communities. There were 2030 patients in wich neurocysticercosis was diagnosed. The majority $(47,6 \%)$ was between the ages of 20 and $40 ; 886(43,7 \%)$ were male, and 1144 (56,3\%) were female. Only 85 patients were from rural communities. Among the appearances we found, there were 983 single calcifications $(48,4 \%), 873$ multiple calcifications $(43,0 \%), 199$ cysts or vesicles $(9,8 \%)$ and 121 granulomas (5,9\%). Hydrocephalus was present in 135 exams $(6,7 \%)$, and in 86 exams there were parasites in two or more evolutional phases. The results are discussed based on the available literature.

KEY-WORDS: Computed tomography; Neurocysticercosis. 


\section{REFERÊNCIAS BIBLIOGRÁFICAS}

ALMEIDA-PINTO, J.; VEIGA.PIRES, J.A.; STOCKER, A.; COELHO, T.; MONTEIRO, L. Cysticercosis of the brain: the value of tomography. Acta Radiologica, v. 29, n. 6, p. 625-628, 1988.

ANTONIUK, S.A.; BRUCK, I.; WITTIG, E.; ACCORSI, A. Neurocisticercose na infância: II. Tomografia computadorizada de 24 pacientes em relação ao tratamento sintomático e com praziquantel. Arq. Neuro-Psiquiat, v. 49, n. 1, p. 47-61, 1991.

ARRUDA, O.A.; CAMARGO, N.J.; COELHO, C.C. Neurocysticercosis: an epdemiological survey in two small rural communities. Arq. Neuro-Psiquiat., v. 48, n. 4, p. 415-420, 1990.

BYRD, S.E.; LOCKE, G.E.; BIGGERS, S.; PERCY, A.K. The computed tomographic appearance of cerebral cysticercosis in adults and children. Radiology, v. 144, p.819-823,1982.

CHEQUER, R.S.; VIEIRA, V.L.F. Neurocisticercose no estado do Espírito Santo. Arq. Neuro-Psiquiat, v. 48, n. 4, p. $431-439,1990$.

GUERREIRO, M.M.; FACURE, N.O.; GUERREIRO, C.A.M. Aspectos da tomografia computadorizada craniana na neurocisticercose na infáncia. Arq. Neuro-Psiquiat, v. 47, n. 2, p. 151-158, 1989.

JOZZOLINO, D.A. Aspectos regionais da neurocisticercose: incidência e avaliação pela tomografia computądorizada em 1000 exames consecutivos. Rev. Imagem, v. 6, n. 1, p. 23-26, 1984.

MACHADO, L.R.; NOBREGA, J.P.S.; BARROS, N.G.;
LIVRAMENTO, J.A.; BACHESCHI, L.A.; SPINA-FRANÇA, A. Computed tomography in neurocysticercosis. Arq. Neuro-Psiquiat, 414-418,1990.

MERVIS, B.; LOTZ, J.W. Computed tomography (CT) in parenchymatous cerebral cysticercosisv. Clinical Radiology, 31, n. 5, p. 521-528, 1980.

MINGUETTI, G.; FERREIRA, M.V.C. Computed tomography in neurocysticercosis. Journal of Neurology, Neurosurgery, and Psychiatry, vo 46, no 10, p. 936-942, 1983.

MIRANDA, A. Neurocysticercosis. American Family Physician, n. 5, p. 1193-1197, 1993.

NASH, T.E.; NEVA, F.A. Recent advances in the diagnosis and treatment of cerebral cysticercosis. Now Engl.J.Med, v. 311, n. 6, p. 1492-1496, 1984.

RAWLINGS, D; FERRIERO, D.M.; MESSING, R.O. Early CT reevaluation after empiric praziquantel therapy in neurocysticercosis. Neurology $v_{0} v_{0} 39_{0} n_{0} g_{0}$ p. $739-740,1989$.

SCHARF, D. Neurocysticercosis: two hundred thirty eight cases from a California hospital. Arch. Neurol., ४. 45, n. 7, p. $777-780,1988$.

SOTELO, J.; GUERRERO, V.; RUBIO, F. Neurocysticercosis: new classification based on active and inactive forms. Arch. Interm. Med., vo 45, no 3, p. 442-445, 1985 。

TAKAYANAGUI, O.M. Neurocisticercose: I. Evolução clinieg laboratorial de 151 casos. Arq. Neuro.Psiquiat, $y_{0} 48$, n. 1, p. 1-10, 1990. 